Perinatal lessons from the past

\title{
Baron Dupuytren (1777-1835) and congenital dislocation of the hip
}

\section{P M DUNN}

University of Bristol, Southmead Hospital, Bristol

Guillaume Dupuytren was one of the ablest doctors in France during the early part of the 19th century. He became surgeon-in-chief at the Hotel Dieu in Paris in 1815. His particular interest was in children's fractures, though perhaps he is best known for his description in 1832 of the contracture of the palmar aponeurosis that was named after him. However, in retrospect, probably his most important contribution was in relation to congenital dislocation of the hip. Although this condition had been discussed in Hippocratic times and mentioned occasionally by other writers, it is to Dupuytren that we owe the first modern account of its anatomy and pathology, its clinical presentation, aetiology, differential diagnosis, and treatment, as the following extracts reveal. ${ }^{12}$

'There is a species of displacement of the upper extremity of the femur, of which I have not found any mention in authors ... This displacement consists in a transposition of the head of the femur, from the cotyloid cavity on to the external iliac fossa (dorsum) of the ilium, a transpostion which exists at birth, and which appears due to a defect in the depth or completeness of the acetabulum, rather than to an accident or disease ... which I shall name original or congenital dislocation . . . If a practitioner is called in at an early period, to see an infant thus affected, certain indications of this deformity may be detected immediately after birth . . . but, as it almost always happens that any vicious conformation, and the infirmities that spring therefrom do not attract the attention of the parents until the child first attempt to walk, it is at this period that surgeons are, in most instances, called upon to establish the existence of the defect. Then the little patients experience considerable difficulty in walking or running, or even in sustaining the weight of the body in the upright posture; but sometimes it even happens that parents, from carelessness or inattention, imagine that their children are only backward

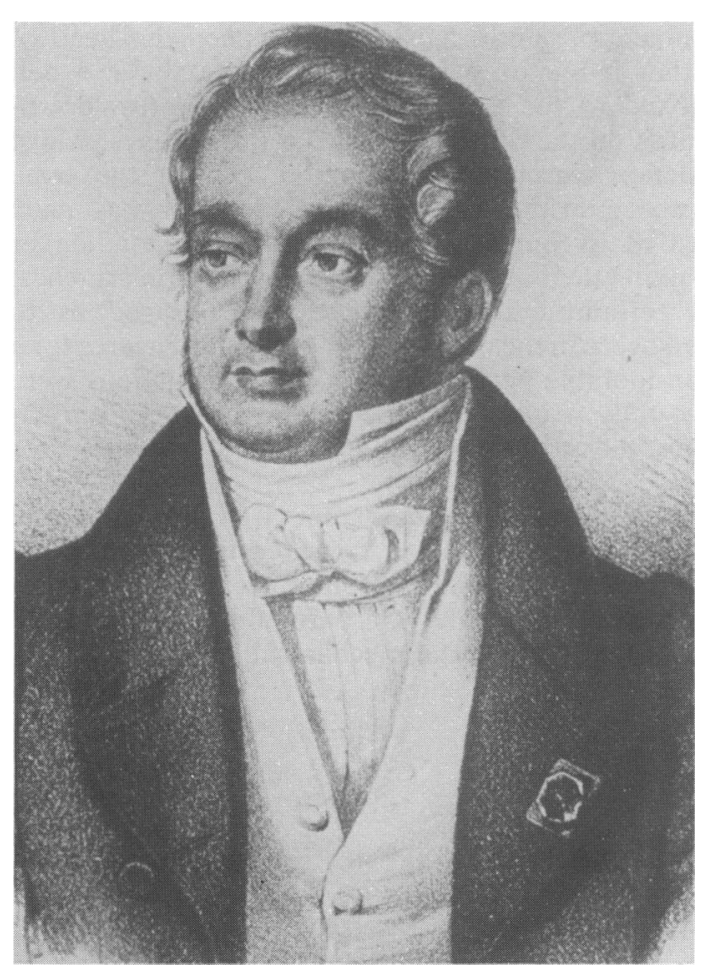

Figure Baron Guillaume Dupuytren of Paris, 1777-1835.

in walking, and do no discover the deformity until three or four years have elapsed.'

Dupuytren's classic description of the clinical presentation is remarkably complete and includes the upward and outwards displacement of the head of the femur, the shortening of the affected limb, the prominence of the greater trochanter, the characteristic lumbar lordosis, the telescoping sign, the 
absence of pain on manipulation, and the distinctive gait. In describing a 9 year old girl with bilateral dislocation he wrote:

'The curvature of the loins, the prominence of the gluteal regions, and the swinging motion of her body when she walked, put me in mind of the waddling of a duck: independently of this, she had very little stability either in standing or walking'.

His attempted treatment of this child is also of great interest:

'The facility with which the limbs could be elongated suggested to us the idea of maintaining such an amount of gentle tension, as to keep the head of either femur on a level with the acetabula: a belt affixed to the hips was employed to press downwards on the upper ends of the thigh bones, whilst traction was employed on the feet for the same purpose, at the same time that the trunk was made fast at its upper part to the bed: in standing or walking the body was always supported on crutches. A certain amount of success encouraged us to persevere in the employment of these measures for a considerable period; but, as our anticipations were not fully realised and the patient was very intractable, the treatment was ultimately abandoned'.

In discussing aetiology Dupuytren dismissed fetal hip disease as a cause, considering abnormal development or actual malformation of the acetabulum more probable. Of particular interest is his discussion of prenatal mechanical factors:
'The position of the lower extremities of the foetus in utero is such, that the thighs are very much bent on the belly, from which it follows that the heads of the thigh bones are continually pressing against the lower and back part of the capsular ligament; a circumstance which, though without effect in wellformed individuals, might, I apprehend, have an injurious influence in such as are weak, or of lax, unresisting fibre. If this premise is conceded, there is not much difficulty in imagining that dislocation may result; and the supposition is further strengthened by the fact that the most powerful of the muscles surrounding the articulation have a constant tendency to draw upwards the heads of the thigh bones, as soon as they escape from the acetabula'.

Dupuytren remarked that congenital dislocation of the hip was 'not so rare as maybe supposed' and that it was frequently bilateral. Furthermore he pointed out that: 'almost all the individuals who are affected with this deformity are females', adding, 'I trust that the observations of others may, at some future period, furnish an interpretation of this phenomenon, and complete that which I have failed to establish in the history of this singular affection'.

\section{References}

1 Dupuytren G. Memoire sur un deplacement original ou congenital de la tete des femurs. Paris: Academie de Medicine, 1926.

2 Dupuytren G. Injuries and diseases of bones. Original or congenital displacement of the heads of the thigh-bones. Translated into English by F leG Clark. London: Sydenham Society, 1847:165-86. 